\title{
Zwischen Geschichtsunterricht und Auslandsvorbereitung: Landeskunde-Unterricht an spanischen Universitäten
}

\author{
Elisabeth F. Basteck
}

\section{Vorbemerkung}

Während sich die theoretische Diskussion innerhalb Deutschlands um landeskundliche Lehr- und Lernkonzepte mit prototypischen Lernergruppen und veränderten Lernzielen auseinandersetzt, kämpft der Landeskundeunterricht in vielen Ländern oft noch mit traditionellen und veralteten Bildungskonzepten für die landeskundliche Lehre innerhalb und außerhalb des Fremdsprachenunterrichts. Dies betrifft besonders die philologischen Studiengänge und damit die internationale Deutschlehrerausbildung. Der Motivationsfaktor, den Landeskunde innerhalb des deutschen Philologiestudiums und Fremdsprachenunterrichts darstellen kann, wird dadurch immer noch zu wenig dafür eingesetzt, Deutschunterricht/Deutschlandstudien an sich interessant und lebendig zu machen.

Am Beispiel der Landeskundelehre an spanischen Universitäten habe ich das Spannungsverhältnis zwischen traditionellem Bildungsrahmen und heutigen Lernerwartungen der Studierenden nachverfolgt. Die Ergebnisse meiner Untersuchung verstehe ich als exemplarisch für die Problematik des Landeskundeunterrichts in vielen Ländern mit traditionellen Lehr- und Lernmethoden (z. B. in Spanien, Portugal, Italien, Griechenland, ...).

Im folgenden werde ich kurz die Situation des Deutschunterrichts und der Landes- kundelehre in Spanien skizzieren. Anschließend werde ich die von mir durchgeführte, empirische Studie mit ihrer Zielsetzung, ihren Ausgangsbedingungen und ihren Ergebnissen vorstellen. Den Abschluß bildet ein Ausblick auf mögliche Konsequenzen für einen landeskundlichen Unterricht, der auf die Bedarfsmeldungen der Studierenden eingeht.

2. Strukturelle und institutionelle Bedingungen der DaF- und LandeskundeLehre in Spanien

Die Situation der Lehre der deutschen Sprache ist in Spanien wenig einheitlich und schwer überschaubar, da nur ca. $45 \%$ des Bildungsbereichs in zuverlässigen Zahlen erfaßt ist. Das Lehr- und Lernangebot der deutschen Sprache läßt sich nach Jané Carbó/Wolff (1991: 204 ff.) grob in 3 Säulen aufteilen:

1. das Schulwesen, in dem das Fach Deutsch sehr schwach vertreten ist;

2. die Universitäten, in denen das Studienfach »Deutsche Philologie« zwar etwas stärker ist, aber erst seit 1952 existiert;

3. der Bereich der Erwachsenenbildung, in dem am meisten Deutsch als Fremdsprache unterrichtet wird.

Ein großes Problem für die spanische Germanistik besteht darin, daß seit den 50er Jahren die meisten Deutsch-Interessenten in Privatinstitutionen ausgebildet werden (vgl. Palau-Ribes 1981: 103), dort die Zah-

Info DaF 31, 1 (2004), 29-51 
len von Lehrkräften sowie Lernern und Lernerinnen aber am wenigsten erfaßt sind und über Methoden und Lernformen keine Informationen bekannt sind. Wie neuere Untersuchungen bestätigen (Orduña 2002: unveröffentlicht), spielt der private Bildungssektor in Spanien von der Primarschule bis zur Universität eine groBe Rolle, und das mit wachsender Tendenz (Zimmermann 2002: 55).

Das öffentliche Schulwesen Spaniens garantiert bis heute nur den Unterricht einer Fremdsprache und dies ist bevorzugt Englisch (vgl. Bosch Roig 1999: $41 \mathrm{ff}$.). Darüber hinausgehende Angebote an Fremdsprachenunterricht werden der Organisation/Direktion der einzelnen Schulen überlassen. Diese müssen allerdings Stellen für Lehrkräfte beim Erziehungsministerium beantragen und den Bedarf dafür nachweisen. Aus diesem Grund fällt es privaten, dezentral organisierten Schulen leicht, u. a. durch ein »erweitertes « Fremdsprachenangebot mit den öffentlichen Schulen zu konkurrieren. Nach Keim (2001: 1516-1517) gilt Deutsch in Spanien (je nach (Touristen-) Region und regionaler Schultradition) als klassische 2. oder 3. Fremdsprache.

Landeskunde wird an den öffentlichen und privaten Primar- und Sekundarschulen Spaniens im Rahmen des Sprachunterrichts unterrichtet. Die dort eingesetzten Lehrwerke werden seit den 60er Jahren fast ausschließlich aus Deutschland importiert (eine unglaubliche Erfolgsgeschichte verzeichnete dabei das Lehrwerk Themen bzw. Themen neu des Hueber Verlags), wodurch indirekt auch ein Methodenimport stattfindet. Im Bereich der Erwachsenenbildung existieren nur Angaben über die öffentlichen Escuelas
Oficiales de Idiomas (EOIs), staatliche Sprachschulen für Erwachsene, in denen ebenfalls (durch die aus Deutschland übernommenen Lehrwerke) landeskundlicher Unterricht in den Sprachunterricht integriert ist.

Eine Ausnahme bildet das landeskundliche Lehrangebot der Universitäten innerhalb des Germanistik- und Dolmetscher/Übersetzerstudiengangs. Für beide Studienfächer (in Spanien wird laut Studienordnung nur ein Fach studiert) schreibt das Erziehungsministerium, Ministerio de Educación i Deporte (MEC), ein 4-stündiges Semesterseminar (mindestens 8 creditos) vor: »Historia y Cultura Alemana. Aspectos geograficos, históricos, ártisticos y culturales" (Deutsche Geschichte und Kultur. Geographische, historische, kulturelle Aspekte und solche aus der Kunst; Übersetzung E. F. B.).

Eine exemplarische Untersuchung verschiedener Studienordnungen ${ }^{1}$ hat gezeigt, daß die Vermittlung landeskundlicher Studieninhalte entsprechend dieser ministeriellen Vorgabe zwischen 1,25\% und 2,94\% der Gesamtstudienzeit der Philologiestudien einnimmt, was weder den offiziellen Darstellungen innerhalb der spanischen Germanistik (zuletzt: Soliño Pazó 2000: 95) noch einer Dreiteilung des Fachs Deutsch als Fremdsprache nach Weinrich entspricht (Weinrich 1980: 44; vgl. auch die Untersuchung von Henrici/Koreik (1994), bei der an deutschen Hochschulen in Magister-Vollzeitstudiengängen für $\mathrm{DaF}$ 15,5\% landeskundlich-kulturkundliche Lehrangebote neben $15 \%$ literaturwissenschaftlichen ermittelt wurden).

Inhaltlich scheint auf den ersten Blick die ministerielle Vorgabe allgemein gehalten

1 Es handelt sich um die Studienordnungen folgender Universitäten: Universitat de Barcelona, Universidad Complutense de Madrid, Universidade de Santiago de Compostela, Universidad de Sevilla, Universitat de Valencia und Universidad de Valladolid. 
zu sein und den einzelnen Universitäten viel Spielraum einzuräumen. Bei genauerem Hinsehen zeichnet sich jedoch das Lernkonzept aus der klassischen Fremdsprachentradition, den lenguas clásicas, ab, die 1845 in das spanische Schulsystem aufgenommen wurden (Ley Pidal) und seither für alle Fremdsprachen als (methodisches und logisches) Vorbild gelten (Marizzi 2002: 63). Im Mittelpunkt steht der klassische Wissenskanon, der sich an historischen, kunsthistorischen, geographischen Wissensinhalten einschließlich der sog. Institutionenkunde orientiert (vgl. z. B. Blanco-Camblor 2000: 261-262; Regales 1992: 349 ff.).

Das ministerielle Landeskundekonzept geht also von dem bildungsbürgerlichen Modell der »Kulturkunde« der 20er Jahre aus (vgl. Melde 1987: 2 ff.), in dem Fremdsprachenkenntnisse vorwiegend einen persönlichen Bildungs- und Statuswert für die Studierenden besitzen. Der traditionelle Allgemeinbildungsanspruch universitärer Ausbildung in Spanien läßt auch bis heute Studienordnungen angefüllt mit altphilologischen Pflichtveranstaltungen, Seminaren über die spanische Sprache und Literatur, Geschichte der Philosophie etc. (vgl. z. B. die Studienordnung der Universidad de Valladolid, in: Regales 1987: 68).

In der spanischen Germanistik werden kulturkontrastive und interkulturelle Ansätze aus der deutschen Fachdiskussion, die z. B. landeskundliche Wissensvermittlung mit kommunikativer Zielsetzung anstrebt, als »Zugabe « zu dem gewaltigen enzyklopädischen Wissenspensum verstanden. Vor dem Anspruch, auch das DACH-Konzept im spanischen Landeskundeunterricht programmatisch umzusetzen, kapitulieren dann jedoch sogar die ehrgeizigsten Studienentwürfe (Blanco-Camblor 2000: 260-261).

Der universitäre Landeskundeunterricht innerhalb der spanischen Germanistik sowie in den Übersetzer-/Dolmetscherstudiengängen kämpft zudem mit einem weiteren schwerwiegenden Problem: die meisten StudienanfängerInnen beginnen ihr Fachstudium mit keinen oder geringen sprachlichen Vorkenntnissen. Saalbach faßt die Lehrsituation wie folgt zusammen:

»Einerseits betrachtet es die Universität nicht als ihre primäre Aufgabe, sich quasi wie eine Sprachschule um die Vermittlung von Fremdsprachenkenntnissen zu kümmern, andererseits muß sie aber die Tatsache berücksichtigen, daß die Fremdsprache für ihre Studenten ein wichtiges Arbeitsinstrument, in den Fremdsprachenphilologien das wichtigste überhaupt, darstellt, sie also in gewisser Weise geradezu dazu verpflichtet ist, dieses Instrument bereitzustellen bzw. wenigstens dazu beizutragen, daß ihre Studenten es sich zu eigen machen können. Das allgegenwärtige Problem des Zeitmangels schlägt sich auch zu Buche: Wo Studiengänge immer weiter verkürzt und in ein reglementiertes Korsett aus knapp bemessener Regelstudienzeit und Höchststudiendauer gezwängt werden, muß man sich sehr wohl überlegen, wie viele Veranstaltungsstunden man der Sprachvermittlung zur Verfügung stellen kann, ohne daß dadurch die Substanz der eigentlichen Studieninhalte allzu sehr zusammenschrumpfen würde. [...] Die Veranstaltungen zur Literatur werden dementsprechend, weitgehend unter Verwendung von Übersetzungen, auf spanisch abgehalten." (Saalbach 1999: 327-328)

Im Gegensatz zum Literaturunterricht ist die Materialienlage spanischsprachiger (Überblicks-)Texte für den Landeskundeunterricht spärlich: es existieren nur zwei spanischsprachige Ganzschriften über die deutsche Kultur der letzten Jahrhunderte (Fulbrook 19901; Janés 1992²),

1 Der dargestellte Zeitraum reicht vom Mittelalter bis 1990.

2 Es wird ein Zeitraum vom Altertum/Mittelalter bis ins Dritte Reich zusammengefaßt. 
wobei nur bei Fulbrook, die 1995 aus dem Englischen ins Spanische übersetzt wurde (!), auf 66 Seiten die Zeit von 1945 bis 1990 überhaupt behandelt wird. 1997 erschien zudem ein Vocabulario de cultura alemana, dessen Sammlung an Stichworten zu geographischem, literarischem und historischem Überblickwissen über deutschsprachige Länder genau jenen traditionellen Kulturbegriff bestätigt, der Landeskundeunterricht zur unbewältigbaren Wissensansammlung macht (Sainz Lerchundi/Sánchez Hernández 1997: 10).

Landes- oder kulturkundlicher Wissenserwerb über die deutschsprachigen Gegenwartsgesellschaften (DACHL) kann also innerhalb des Philologiestudiums nur über den (vorangegangenen erfolgreichen) Erwerb der deutschen Sprache erfolgen. Von einer Fähigkeit, authentische Publikationen der Gegenwart (wie tagespolitischer Pressemitteilungen, historischer Texte etc.) zu lesen, kann bei den Studierenden der ersten Studienjahre, in denen die meisten Landeskundeseminare vorgeschrieben sind, nicht die Rede sein.

Der explizite Landeskundeunterricht steht also in der Spannung zwischen Sprachunterricht, der sich in Spanien bis heute stark an der Grammatikvermittlung orientiert, und kognitiver Wissensvermittlung in der spanischen Muttersprache. Interkulturelle, anthropologische Landeskunde (nach Geertz 1983, vgl. z. B. Penning 1995) oder Ansätze zu Culture/Language Awareness (z. B. Knapp-Potthoff 1997, Kramsch 1991 etc.) oder politischer Bildung (z. B. Doyé 1992) finden also, wenn überhaupt, innerhalb des deutschen Fremdsprachenunterrichts statt, vorausgesetzt, daß moderne Ansätze aus importierten DaF-Lehrwerken aufgegriffen werden. Die (zarte) Landeskunde-Diskussion innerhalb der spanischen Germanistik mündet angesichts dieser Grundsatzproblematik häufig in
Vorschlägen zu Lesetechniken (vgl. Regales 1992, Bachmann 1992, Blanco-Camblor 2002) und beschäftigt sich selten mit der Curricular-Frage: Was soll, was kann unter den oben genannten Bedingungen im universitären Landeskundeunterricht vermittelt werden?

\section{Die Befragung der Studierenden an 12 spanischen Universitäten}

\subsection{Ausgangsbedingungen der Befra- gung}

Als DAAD-Lektorin an der Universidad de Salamanca (1997-1999) und der Universitat de Barcelona (1999-2002) hatte ich (wie die meisten LektorInnen auf der Iberischen Halbinsel) die Aufgabe, neben Sprache auch Landeskunde zu unterrichten. Dabei war die Spannung zwischen (großem) inhaltlichem Interesse und sprachlicher Barriere bei den Studierenden im Unterricht permanent präsent. Bei meiner Suche nach adäquaten, neuen Lehrformen innerhalb des traditionell organisierten Lehrbetriebs an der spanischen Universität entwarf ich mit meinem Kollegen Prof. Dr. Jesus Hernandez Rojo an der Universidad de Salamanca einen Befragungsbogen für Studierende landeskundlicher Seminare. Uns ging es darum, Informationen über den Landeskundeunterricht an spanischen Universitäten allgemein $\mathrm{zu}$ sammeln und Bildungskonzepte für spanische Studierende zu suchen, die deren Bedürfnissen entsprechen. Dazu muß erwähnt werden, daß die europäischen Studenten-Austauschprogramme (das ERASMUS- bzw. das SOKRATES-Programm) seit ihrer Einführung zu Beginn der 90er Jahre an spanischen Universitäten einen durchschlagenden und anhaltenden Erfolg haben und heute ein mindestens 3-monatiger Aufenthalt in einem deutschsprachigen Land zum fast selbstverständlichen Teil des Philologiestudiums gehört. Diese 
neue Ausrichtung auf einen Auslandsaufenthalt mit seinen Konsequenzen für studentische Erwartungen, Bedürfnisse und anschließend veränderte Perspektiven bezüglich des Studiums wurden bisher nie untersucht. Wie ich im folgenden zeigen werde, betreffen diese neuen Entwicklungen den Landeskundeunterricht aber in besonderem Maße.

\subsection{Ziele und Methoden der Befragung}

Bei der Befragung interessierte auf inhaltlicher Ebene, welche Interessen die Studierenden allgemein haben und welche spezifisch auf Deutschland gerichteten Interessen sie in ihrem Studium verfolgen. Welche Rolle spielt dabei z. B. die traditionelle Vorstellung von Landeskunde $=$ Geschichtsunterricht? Es wurde bei der Untersuchung auch versucht, die spanische Übersetzung des Begriffs "Landeskunde" in cultura, civilisación oder sociedad in verschiedene Begriffsbereiche aufzusplittern. Die grundsätzliche Frage dabei war: verschiebt eine veränderte Wortkonnotation auch die Erwartungen an das Fach?

Auf methodischer Ebene wurde gefragt, ob die kognitiven Lernmethoden den Fähigkeiten und Erwartungen der Studierenden entsprechen und welche Lernformen und Informationsquellen sie bevorzugen, um Wissen über das Zielsprachenland zu erhalten. Zusätzlich wurde auch nach dem Effekt des landeskundlichen Wissenserwerbs auf das Studium der deutschen Sprache überhaupt gefragt.

Die Befragung wurde zwischen September 2001 und Oktober 2002 an insgesamt 12 spanischen Universitäten unter Studierenden der Germanistik, Übersetzungs- und Dolmetscherwissenschaft und der deutschen Sprache in Form eines Fragebogens mit 14 Fragen durchgeführt. Dank zweier landesweiter Mailing-Listen $^{1}$ schrieb ich per E-Mail alle Germanistik- und Übersetzer-/Dolmetscherabteilungen an spanischen Universitäten an. Mein Anschreiben wurde unterstützt durch die in Spanien wichtige Mund-zuMund-Propaganda befreundeter Kolleginnen und Kollegen. Auch dank der Unterstützung der DAAD-LektorenkollegInnen war der Rücklauf umfassend genug, um repräsentativ zu sein: an der Befragung nahmen insgesamt 282 Studierende von 12 Universitäten teil (siehe Abb. 1).

Außer an der Universidad de Sevilla und der Universitat de Vic handelte es sich bei allen befragten Gruppen um TeilnehmerInnen an ausschließlich landeskundlichen Seminaren über deutsche bzw. deutschsprachige Länder (explizite Landeskunde). Die große Teilnehmerzahl an der Universidad de Malaga, Fakultät Traducción e Interpretatión, geht auf die Befragung von insgesamt drei Seminargruppen von drei Studienjahren zurück:

1. Studienjahr: 46 Befragte;

2. Studienjahr: 32 Befragte;

3. Studienjahr: 25 Befragte.

Der Befragung lag die methodische Konzeption der quantitativen, nicht-experimentellen Forschung zu Grunde: von den Studierenden sollten möglichst viele vorgegebene Antworten angekreuzt und nur bei insgesamt 3 von 14 Fragen eine eigene Bewertungen (qualitative Forschung) gegeben bzw. individuelle Standpunkte erläutert werden. Bei fast allen geschlossenen Fragen wurde den Befragten auch die Möglichkeit gegeben, neben den vorgegebenen Antworten ei-

1 von Prof. Dr. Anton Haidl, Universidad de Cádiz, und Sophie Caesar, Hueber-Verlag/ Editorial Idiomas, Madrid, denen ich beiden an dieser Stelle meinen Dank aussprechen möchte. 
Abb.1: An der Befragung beteiligte Universitäten

(die Zahl in Klammern gibt die Zahl der Fragebögen an, die Prozentzahl gibt die Anteile zur Stichprobengröße von 282 Fragebögen an, jeweils auf- bzw. abgerundet)

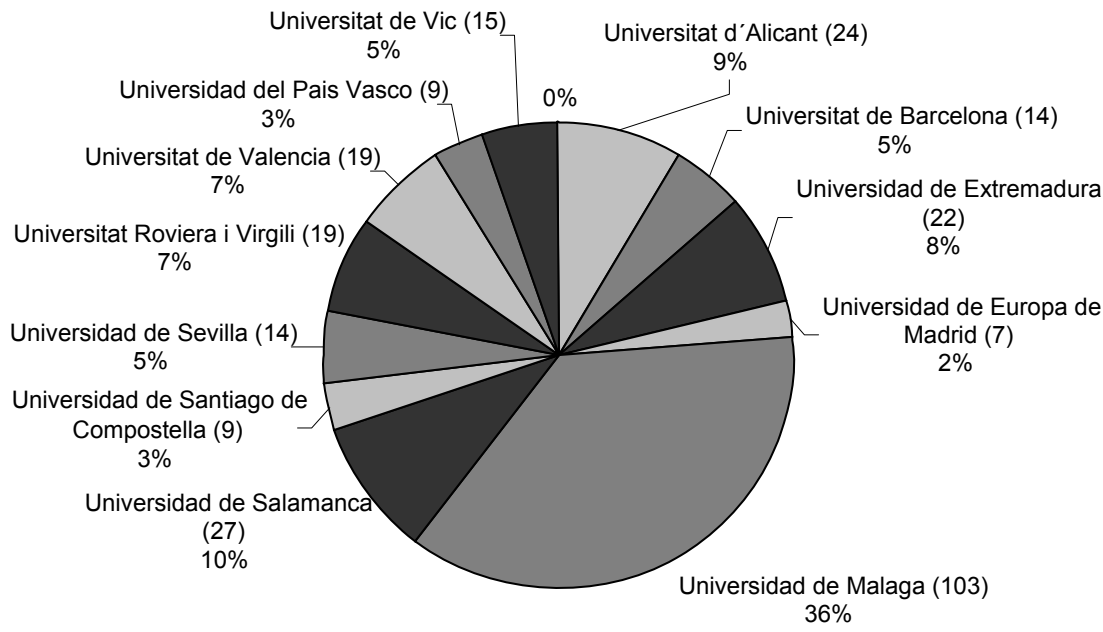

gene Meinungen zu ergänzen. Dieses Angebot wurde insbesondere bei den Fragen 6, 10 und 12 von den Studierenden häufig wahrgenommen (Fragebogen: siehe Anhang). Die folgende Darstellung gibt eine stark gekürzte Auswertung der Untersuchung wieder, bei der aus methodischen Gründen die Fragen 6 und 9 herausgenommen werden mußten ${ }^{1}$.

\subsection{Ergebnisse der Umfrage}

\subsubsection{Die Interessengebiete der Studierenden}

Nach Klärung der institutionellen Einordnung der evaluierten Seminargruppen konzentriert sich der erste Teil des
Fragebogens auf Anfragen nach den Interessen der Studierenden im allgemeinen (in ihrem Herkunftsland, im alltäglichen Bereich).

Frage 4:

Welche Themen interessieren Dich im Allgemeinen? (¿Qué temas te interesan en general?).

Im 2. Schritt wurde nach den Interessen bezüglich der Fremdkultur Deutschland gefragt:

\section{Frage 5:}

Welche dieser Themen interessieren Dich in Bezug auf Deutschland? (¿Cuáles de estos temas te interesan con relación a Alemania?). Für die Auswertung wurden die

1 Eine umfassende Auswertung wird im Rahmen meiner Dissertation publiziert, die unter dem Titel Landeskundeunterricht an spanischen Universitäten im Sommer 2004 erscheint. 
Differenzen gegenübergestellt. Die angebotenen Antwortmöglichkeiten (bei beiden Fragen die (fast) gleichen 23) wurden thematisch einander zugeordnet (vgl. Tabelle Abb. 2).

Die vorgeschlagenen Antworten waren eine Zusammenstellung unterschiedlichster Bereiche, die sich aus der Analyse mehrerer spanischer Jugendzeitschriften (Bravo u.a.) ergeben hatten und mit den im Studium üblichen, klas- sischen landeskundlichen Themen (Geographie, Geschichte, Politik, Parteien, Literatur/Romane, Fachliteratur über ..., Die Europäische Union, Gewerkschaften) vermischt wurden. Die Studierenden wurden auch aufgefordert, eigene Themen zu ergänzen, was allerdings nur vereinzelt geschah (insgesamt in 13 von 1482 Nennungen bei Frage 4 und in 10 von 1368 Nennungen bei Frage 5$)^{1}$.

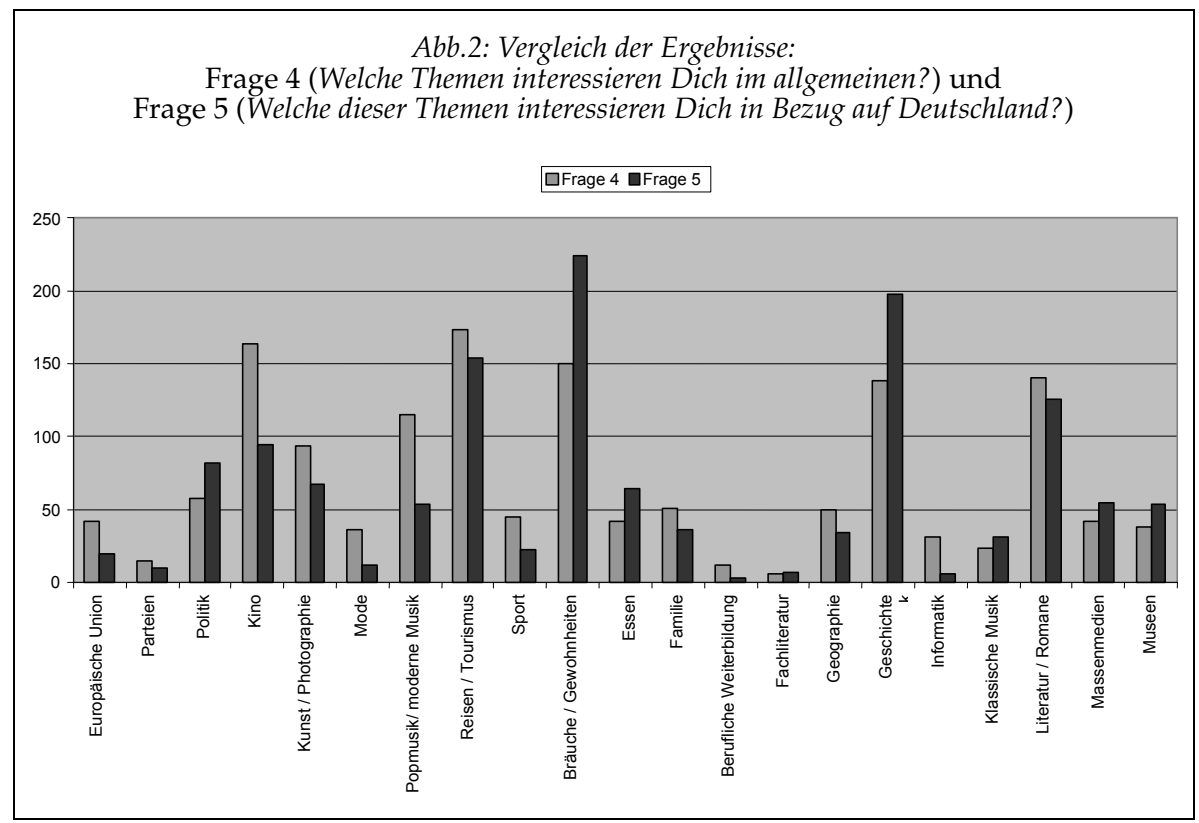

1 Ergänzt wurden bei Frage 4 die Themen (in alphabetischer Reihenfolge) durch Einzelnennungen von »filosofía «; »ideologia/religion; »naturaleza/medioambiente «; »naturaleza«; »ONGs/medioambiente«; »sociología «; »modos de vida/mentalidad «; »báile«; »amor«; »educación«; »psicología«; »idiomas«; »ensayos sobre temas actuales«; und »Volkskunde«. Bei Frage 5 wurden folgende Themen ergänzt (in alphabetischer Reihenfolge): »arquitectura«; »báile«; »derecho«; »dialectos«; »educación«; »filosofía« (2x); »ideología«; »tradiciones«; »Theater«. Insgesamt hat sich das im Fragebogen angewandte Verfahren als ergiebig gezeigt, die Befragten zum Formulieren der eigenen, individuellen Lernsituation mit einer Leerzeile bzw. weiterführenden Pünktchen aufzufordern. Z. B. bei Frage 5, wo bei dem Punkt »Literatura especializada...« das Thema »teatro« (Fachliteratur über das Thema Theater) und »en general« (im allgemeinen) ergänzt wurde. 
Wie die Tabelle zeigt, konzentrieren sich die Interessensgebiete der Studierenden an den befragten Universitäten trotz unterschiedlicher Studienschwerpunkte und Studienordnungen sowie regionalen Unterschieden auf einige wenige Gebiete, am meisten auf den Bereich »Bräuche und Gewohnheiten « bezüglich des Interesses an Deutschland und »Reisen/Tourismus « bezüglich der Allgemeininteressen, was sich durch die Zielgruppe, nämlich Studierende einer Philologie, erklären läßt.

Obwohl es teilweise nicht einfach war, die 23 gegebenen sowie freien Antworten der Studierenden eindeutig abzugrenzen (z.B. das einmal ergänzte "amor«), mußten die Antworten für die Auswertung thematisch gebündelt werden. Dabei wurden vier Themengebiete gebildet:

1. Themen des Freizeitbereichs insgesamt (Kino, Kunst/Photographie, Mode, Popmusik/moderne Musik, Reisen/ Tourismus, Sport, zwei Einzelnennungen): bezüglich Spanien/allgemein: $42 \% \Leftrightarrow$ in Bezug auf Deutschland: $29,3 \%$

2. Bildungsbereich insgesamt (Berufliche Weiterbildung, Fachliteratur, Geographie, Geschichte, Informatik, Klassische Musik, Literatur/Romane, Massenmedien, Museen, 4 Einzelnennungen): bezüglich Spanien/allgemein: $32,7 \% \Leftrightarrow$ in Bezug auf Deutschland: $37,5 \%$

3. alltagskultureller Bereich (Bräuche/ Gewohnheiten, Essen, Familie, zwei Einzelnennungen): bezüglich Spanien/allgemein: $16,3 \% \Leftrightarrow$ in Bezug auf Deutschland: $23,6 \%$

4. politischer Bereich insgesamt (Die Europäische Union, Parteien, Politik, Gewerkschaften, ONGs/medioambiente): bezüglich Spanien/allgemein: $7,8 \% \Leftrightarrow$ in Bezug auf Deutschland: $8,2 \%$
Die Anzahl der Nennungen überwiegt eindeutig bei den Themen des Freizeitbereichs insgesamt, was sich u.a. auf das durchschnittliche Alter der spanischen Studierenden (zwischen 18 und 22 Jahren) zurückführen läßt. Hier fällt allerdings auf, daß auch ein deutliches Interesse am deutschen Freizeitbereich vorhanden ist, der in der traditionellen Konzeption von Landeskunde in den Studienordnungen nicht vorgesehen ist. Im Bildungsbereich insgesamt unterscheiden sich die Nennungen bezüglich Spanien $(\mathrm{E}=$ $32,7 \%$ ) erstaunlich wenig von denen bezüglich Deutschlands $(\mathrm{D}=37,5 \%)$. Bei genauerer Betrachtung z.B. im Bereich Literatur/Romane überrascht, daß sich die Studierenden hier sogar noch mehr für die Literatur ihres eigenen Kulturkreises bzw. allgemein für Literatur interessieren als für die der Zielkultur. Neben dem klassischen, literarischen Bildungsfach erscheint auch das häufig genannte Interesse an Geschichtswissen (E: 9,3\% $\Leftrightarrow$ D: $14,4 \%$ ) in das traditionelle Verständnis von Landeskunde $\mathrm{zu}$ passen und entspricht früheren Untersuchungen bei französischen Studierenden der Germanistik (vgl. Picht 1980). Dieser zog daraus den Schluss, daß

»die Möglichkeit zur Beschäftigung mit deutschen Themen primär nicht durch das Interesse an den deutschen Zusammenhängen geprägt ist, sondern durch die Traditionen und Systemzwänge spezifischer gesellschaftlicher Situationen im jeweiligen Ausgangsland. Entscheidend ist hierbei vor allem das Prüfungssystem bzw. die Voraussetzungen und Aufstiegsmuster beruflicher Karriere» (Picht 1980: 129).

Um so erstaunlicher erscheint daher das große studentische Interesse am alltagskulturellen Bereich (E: 16,3\% $\Leftrightarrow$ D: 23,6\%), der in der spanischen Germanistik und den Dolmetscher-/Übersetzerstudiengängen laut Prüfungsordnung überhaupt keine Rolle spielt. Am wenigsten interes- 
sierten die Befragten die Themen aus dem politischen Bereich (E: 7,8\% und D: $8,2 \%)$. Das bedeutet eine deutliche Absage an das landeskundliche Konzept sog. »Institutionenkunde«.

Frage 7 stellt zu den Fragen 4 und 5 eine Gegenprobe dar, in der die Lernerfahrungen der Studierenden als Ausgangsbasis für Bedarfsmeldungen an den Landeskundeunterricht genommen werden:

Erinnerst Du Dich, ob Du im Sprachunterricht oder in der Uni schon einmal gedacht hast, daß Du gerne mehr über ein bestimmtes Thema wissen möchtest? Erinnerst Du Dich, $z u$ welchem Bereich dieses Thema gehörte? (¿Recuerdas en clase de lengua o en la universidad haber pensado que te gusteria saber más sobre algún tema en concreto? ¿Recuerdas a qué ámbito pertenecía esta tema?)
Die Studierenden wurden bei dieser Frage zugleich aufgefordert, ihren Wissensbedarf in der Ziel- und der Herkunftskultur selbst einem übergeordneten Themengebiet (Gesellschaft, Bräuche/Gewohnheiten, Essen, Geographie, Politik, Wirtschaft, Geschichte, Kunst, Musik) zuzuordnen. Unter den Antwortmöglichkeiten wurde das Themengebiet »Literatur/Romane« bewußt ausgelassen, da Literaturunterricht im Vergleich zum landeskundlichen in den universitären Studienordnungen viel breiter abgedeckt wird (Philologiestudien werden traditionell als Literaturstudien angesehen). Das Gesamtgebiet "Gesellschaft « wurde zu den angebotenen Antwortmöglichkeiten hinzugenommen (ergänzt wurde nur zwei (Mal) $)^{1}$ :

\section{Abb. 3: Cluster Frage 7}

\begin{tabular}{|l|c|c|c|c||}
\hline $\begin{array}{l}\text { Genannte } \\
\text { Themenbereiche }\end{array}$ & $\begin{array}{c}\text { über Spanien } \\
\text { absolute Zahlen von } \\
\text { insgesamt 664 Nennungen } \\
\text { (282 Befragte) }\end{array}$ & $\begin{array}{c}\text { Prozent- } \\
\text { angaben }\end{array}$ & $\begin{array}{c}\text { über Deutschland } \\
\text { absolute Zahlen von } \\
\text { insgesamt 779 Nennungen } \\
\text { (282 Befragte) }\end{array}$ & $\begin{array}{c}\text { Prozent- } \\
\text { angaben }\end{array}$ \\
\hline Gesellschaft & 75 & $11,2 \%$ & 130 & $16,6 \%$ \\
\hline Bräuche/Gewohnheiten & 75 & $11,2 \%$ & 165 & $21,1 \%$ \\
\hline Essen & 15 & $2,2 \%$ & 27 & $3,4 \%$ \\
\hline Geographie & 41 & $6,1 \%$ & 66 & $8,4 \%$ \\
\hline Politik & 85 & $12,8 \%$ & 69 & $8,8 \%$ \\
\hline Wirtschaft & 63 & $9,4 \%$ & 23 & $2,9 \%$ \\
\hline Geschichte & 137 & $20,6 \%$ & 155 & $19,8 \%$ \\
\hline Kunst & 122 & $18,3 \%$ & 90 & $6,8 \%$ \\
\hline Musik & 50 & $7,5 \%$ & 53 & $11,5 \%$ \\
\hline \hline
\end{tabular}

Wie schon bei Frage $5 \mathrm{zu}$ sehen war, überrascht auch hier das Ergebnis da-

durch, daß die Studierenden offensichtlich im Fremdsprachenunterricht einen

1 Die Ergänzungen bei Frage 7 waren Einzelnennungen von: »Gesellschaft« und »filosofía«. 
neuen Blick auf ihre eigene Kultur erfahren und ihr Bildungsbedarf - bezüglich der »herkömmlichen « landeskundlichen Wissensgebiete wie Geschichte, Kunst und Politik - in ihrer Herkunftskultur größer ist als in der Zielkultur. An der deutschen Kultur fehlten ihnen vorwiegend alltagskulturelle Kenntnisse (usos y costumbres entspricht im Spanischen einer feststehenden Redewendung und läßt sich deutsch etwa mit >Bräuche und Gewohnheiten entsprechend kultur- und organisationsanthropologischen Forschungsmethoden - zwischen zwei Kulturebenen unterschieden werden:

\section{»Kultur:}

1. Mentales Training bzw. mentale Verfeinerung. Zivilisation. [...]

2. Die kollektive mentale Programmierung, die die Mitglieder der einen Gruppe oder Kategorie von Menschen von einer anderen unterscheidet. Diese Bedeutung entspricht dem in der Anthropologie verwendeten Begriff >Kultur $\ll$. (Hofstede 1997: 401)

»)Kultur Zwei< umfaßt nicht nur Tätigkeiten, die den Geist verfeinern sollen, sondern auch gewöhnliche und niedrige Dinge des Lebens: Grüßen, Essen, das Zeigen oder Nichtzeigen von Gefühlen, das Wahren einer gewissen physischen Distanz zu anderen, Geschlechtsverkehr oder Körperpflege.« (Hofstede 1997: 5)

Der studentische Bedarf innerhalb des deutschen Fremdsprachenunterrichts fällt unter der Bezeichnung usos y costumbres also in den von Hofstede genannten alltagskulturellen Bereich »Kultur Zwei«. Dagegen vermißten die spanischen Studierenden Wissen über landeskundliche Themenbereiche, die sich als Trägerbereiche der »Kultur Eins « einordnen ließen (Geschichte, Kunst, Geographie, Musik) zuerst in ihrer eigenen Kultur. Erst an zweiter Stelle fehlte Wissen im »Kultur Eins«-Bereich für die fremde Sprache und Kultur, wobei auch hier die Bedarfs- meldung hoch ist (Geschichte in D: $19,8 \%$, Kunst in D: $11,5 \%$; Musik in D: 6,8\%).

Ausgehend von dieser Beobachtung läßt sich ein neuer Bildungsauftrag an den Fremdsprachen- und Landeskundeunterricht (nicht nur in Spanien) erkennen: Primär hat der Kontakt zu einer anderen Kultur einen Spiegeleffekt auf die eigene Herkunft: die faktischen Grundlagen des eigenen Landes werden in ihrer Bedeutung erkannt und interessant. Bezüglich der Fremdkultur hat Landeskundeunterricht zuerst die Funktion, auf die alltagskulturelle Wirklichkeit im Zielsprachenland vorzubereiten. Wie die weitere Auswertung der Befragung der spanischen Studierenden zeigt, sind die Lernerinnen und Lerner heute auf eine kommunikative Anwendung der Fremdsprache ausgerichtet.

\subsubsection{Bevorzugte Informationsquellen und Lernstrategien der Studierenden}

Auf der Suche nach neuen, alternativen Materialien für den Landeskundeunterricht in Spanien, die nicht auf Vorkenntnissen in der deutschen Sprache zum Verstehen authentischer Texte aufbauen, wurden die Studierenden nach der Glaubwürdigkeit öffentlicher Medien in Spanien mit ihrer Berichterstattung gefragt. Frage 8:

Um neue Information über ein Thema (egal, welches) zu erhalten, welchem öffentlichen Medium glaubst Du am meisten? (bezüglich der Glaubwürdigkeit der vermittelten Information, z.B. bei den Anschlägen in den USA). (Para obtener información nueva sobre algún tema (sin importar cuál), ¿a qué medio de comunicación das más credibilidad? (en cuanto a la fiabilidad de la información transmitida en, por ejemplo, los atentados en los EEUU))

Die Antworten wurden folgendermaßen gegeben: 
Abb. 4: Cluster Frage 8

\begin{tabular}{|l|c|c||}
\hline Welches öffentliche Medium? & $\begin{array}{c}\text { Absolute Zahlen } \\
\text { von insgesamt 582 Nennungen } \\
\text { (282 Befragte) }\end{array}$ & $\begin{array}{c}\text { Prozent- } \\
\text { angaben }\end{array}$ \\
\hline Der Zeitung .. & 80 & $13,7 \%$ \\
\hline Meine Zeitung, die ich immer kaufe & 54 & $9,2 \%$ \\
\hline Einer Fachzeitschrift über das Thema & 88 & $15,1 \%$ \\
\hline Einem Buch von einer/m bekannten AutorIn & 15 & $2,5 \%$ \\
\hline einem wissenschaftlichen Fachbuch & 41 & $7,0 \%$ \\
\hline einer Statistik & 12 & $2,0 \%$ \\
\hline dem TV & 92 & $15,8 \%$ \\
\hline dem Radio & 68 & $11,6 \%$ \\
\hline $\begin{array}{l}\text { Ich glaube es nur, wenn die Informationen bei } \\
\text { verschiedenen Medien übereinstimmen. }\end{array}$ & 129 & $22,1 \%$ \\
\hline
\end{tabular}

Da hier die Möglichkeit bestand, Mehrfachnennung (bis zu 3 Antworten) zu geben, ist die häufigste Nennung von Ich glaube es nur, wenn die Informationen bei verschiedenen Medien übereinstimmen als quasi "sicherste" Antwort zu erklären. Sicher beeinflußte aber auch der Zeitpunkt der Umfrage (zwischen September 2001 und Oktober 2002) das Antwortverhalten: direkt nach dem 11. September 2001 war unter den Antworten deutlich mehr Mißtrauen den Massenmedien gegenüber erkennbar. Deutlich zeigt sich die Neigung zum Fernsehkonsum (in einem spanischen Haushalt läuft der TV üblicherweise den ganzen Tag) im Gegensatz zur Informationsquelle öffentlicher Printmedien (Zeitungen). Erstaunlich ist die Abneigung der Studierenden gegen wissenschaftliche Fachliteratur: Sie ziehen populärwissenschaftliche Zeitschriften vor: der Umgang mit wissenschaftlichen $\mathrm{Pu}-$ blikationen scheint bei den angehenden Akademikerinnen und Akademikern wenig selbstverständlich oder beliebt zu sein. Einzelne Kommentare der Befragten zeigen jedoch auch das Mißtrauen gegenüber den von der spanischen Regierung kontrollierten Informationsquellen (TV):
»Ninguna información en los medios de comunicación es válida para mi al $100 \%$, ni aunque coincida. Todo es relativo y manipulado según el interés «. (Keine Information der Massenmedien ist für mich zu $100 \%$ gültig, auch nicht, wenn sie übereinstimmt. Alles ist relativ und dem Interesse entsprechend manipuliert) oder "No prestar ningún fiabilidad. Es la comerz de informarse«. (Ich schenke keinerlei Glaubwürdigkeit. Es ist der Kommerz zu informieren/sich $\mathrm{zu}$ informieren/informiert zu sein).

In Frage 10 wurde noch genauer nach dem politischen (und nicht historischen) Interesse der Studierenden gefragt, da sich Medienberichte über Deutschland meist auf politische Tagesereignisse beziehen (oft eine Zusammenfassung mehrerer Berichte deutscher Tageszeitungen): In spanischen Zeitungen gibt es viele Informationen über Deutschland und die deutsche Politik. Interessieren Dich diese Artikel? Warum? Warum nicht? (En los periódicos españoles existe mucha información sobre Alemania y la política alemana.¿Te interesan estos articulos? ¿Por qué?¿Por qué no?)

Bei dieser offen gestellten Frage wurden die Kommentare der Studierenden in ei- 
nem Cluster nach positiven (+) und negativen (-) Aussagen geordnet. Grundsätzlich überwiegt eine positive Einschätzung der Medieninformationen mit $59,4 \%$. Dennoch wurden viele negative Stellungnahmen von den Studierenden formuliert. Die meistgenannte Antwort war politisches Desinteresse allgemein »No me interesa la política «. (Politik interessiert mich nicht) oder Zeitmangel "No suelo leer el periodico por falta de tiempo, escucho el telediario". (Ich lese keine Zeitung, weil die Zeit dazu fehlt, ich sehe die TV-Nachrichten). Trotz der positiv formulierten Frage wurde relativ häufig Kritik an zu wenig Informationen über deutschsprachige Länder geäußert und daran, daß die Nachrichten unverständlich seien und ohne Hintergrundinformationen nicht zugeordnet werden könnten: "La verdad es que no hay muchas articulos además aunque interesan muchas veces no me entero muy bien porque hay muchas cosas que no sé«. (Die Wahrheit ist, es gibt nicht viele Artikel, und obwohl sie mich interessieren, verstehe ich oft nicht sehr gut, weil es viele Dinge gibt, die ich nicht weiß.) Hier wird der Mangel an landeskundlichem Hintergrundwissen über die deutschsprachigen Gegenwartsgesellschaften zum Hindernis, spanischsprachige öffentliche Quellen zu verstehen und neue Informationen aufzunehmen. Landeskundeunterricht hätte hier die Aufgabe, gesellschaftliche Grundkenntnisse allgemein im Überblick zu vermitteln, in die die Studierenden Informationen aus Medienberichten u. a. einordnen könnten.

Grundsätzlich dokumentieren zahlreiche Kommentare aber auch das studentische Interesse am gesellschaftlichen Leben in Deutschland: "Si, porque aúnque no me interesan los temas políticas en concreto si la sociedad en general ya que estoy estudiando el idioma«. (Ja, denn obwohl mich politische Themen im einzelnen nicht interessieren, interessiert mich die Gesellschaft im allgemeinen, da ich ja die Sprache studiere/lerne.) Bei einigen Befragten spielt für ihr Interesse auch der internationale wirtschaftliche und politische Einfluss Deutschlands eine Rolle. Der Kontakt zu Deutschland wird wieder zum Spiegel, diesmal für die spanische Außen- und Europapolitik: "Si, porque me interesa la política alemana y como afecta su relación con la política española«. (Ja, weil mich die deutsche Politik interessiert und wie ihr Verhältnis zur spanischen Politik beeinflußt wird) oder " $\mathrm{Si}$, porque considero que actualmente Alemania es la primera potencia económica dentro de la U.E.«. (Ja, weil ich davon ausgehe, daß Deutschland im Moment die erste Wirtschaftsmacht innerhalb der EU ist.).

Eindeutig ist auch bei dieser Frage der Vorbereitungsanspruch auf kommende/ geplante Auslandsaufenthalte deutlich erkennbar: » $\mathrm{Si}$, porque ya estoy estudiando alemán, quiero saber que me encontraré cuando vaya«.« (Ja, weil ich ja Deutsch lerne/studiere, möchte ich wissen, was ich dort vorfinde, wenn ich dorthin fahre.) Eine studentische Antwort erklärt das Interesse vieler Studierender der Philologie an der Historie des Zielsprachenlandes, denn nur sie sei die einzig zuverlässige Informationsquelle: "No creo en la objectividad periodística, creo más en el conocimiento histórico«. (Ich glaube nicht an eine journalistische Objektivität, sondern mehr an historisches Wissen.)

Im nächsten Schritt der Untersuchung wurden die Studierenden nach ihren bevorzugten Lernmethoden gefragt. Frage 11: Wenn Du Dir ein Thema über Deutschland aussuchen könntest, wie würdest am liebsten etwas darüber erfahren? (Si tuvieras que escoger un tema sobre Alemania, ¿como te gustería estudiarlo?). Die Antworten ließen sich wie folgt ordnen: 
Abb. 5: Cluster Frage 11

\begin{tabular}{|l|c|c|c||}
\hline Frage nach gewünschter Arbeitsmethode/Lernform & $\begin{array}{c}\text { Absolute Zahlen } \\
\text { von insgesamt } \\
\text { 489 Nennungen } \\
\text { (282 Befragte) }\end{array}$ & $\begin{array}{c}\text { Prozent- } \\
\text { angaben }\end{array}$ & Arbeitsform \\
\hline $\begin{array}{l}\text { Eine allgemeine Einführung in einem Überblicksse- } \\
\text { minar erhalten (neben anderen Themen) }\end{array}$ & 53 & $10,8 \%$ & Typ A \\
\hline $\begin{array}{l}\text { Einen Vortrag/eine Konferenz von einem Speziali- } \\
\text { sten/einer Spezialistin darüber hören }\end{array}$ & 57 & $11,6 \%$ & Typ A \\
\hline $\begin{array}{l}\text { Ein spezielles Seminar belegen } \\
\text { (1) }\end{array}$ & 15 & $23,5 \%$ & $\begin{array}{c}\text { indifferent, beide } \\
\text { Arbeitsformen } \\
\text { möglich }\end{array}$ \\
\hline $\begin{array}{l}\text { Selbständig das Thema erforschen und eine schriftli- } \\
\text { Ein Buch darüber lesen }\end{array}$ & 47 & $9,6 \%$ & Typ B \\
\hline $\begin{array}{l}\text { Ein Semester frei nehmen und mehrere Bücher dar- } \\
\text { über lesen }\end{array}$ & 25 & $5,1 \%$ & Typ B \\
\hline $\begin{array}{l}\text { In einer Arbeitsgruppe Informationen über das } \\
\text { Thema sammeln und sich dann über das erhaltene } \\
\text { Wissen austauschen }\end{array}$ & 75 & $15,3 \%$ & Typ B \\
\hline $\begin{array}{l}\text { Über das Internet einen deutschen Gesprächspart- } \\
\text { ner/eine Gesprächspartnerin suchen, um mit ihr/ } \\
\text { ihm über das Thema zu sprechen }\end{array}$ & 27 & $5,5 \%$ & Typ B \\
\hline
\end{tabular}

Unterteilt man die in Frage 11 gegebenen Antworten in Arbeitsformen unterschiedlichen Typs, lassen sich zwei Gruppierungen bilden: Typ A, der auf frontale Unterrichtsgestaltung und ausschließlich kognitive Wissensvermittlung ausgerichtet ist, und Typ $B$, bei dem das autonome und selbstverantwortliche Lernen und Forschen die Grundlage des Wissenserwerbs darstellt. Bei den im Umfragebogen vorgeschlagenen 8 Antworten wurde bewußt eine Betonung auf die autonomen Lernformen gelegt. Die Studierenden hatten aber auch hier die Möglichkeit, ihre Antwort offen zu formulieren und zu ergänzen.

Das Ergebnis zeigt: die befragten Studierenden würden am liebsten zweigleisig fahren und sich gerne sowohl frontal und überblicksartig belehren lassen (Eine allgemeine Einführung ... erhalten: 10,8\% und Einen Vortrag/Konferenz ... hören: $11,6 \%)$ als auch völlig selbständig und unabhängig gezielte Themenbereiche er- arbeiten und sich mit anderen Studierenden austauschen (In einer Arbeitsgruppe Informationen ... sammeln...: 15,3\%, Selbständig das Thema erforschen.... 9,6\%, Ein Semester ... mehrere Bücher ... lesen: 5,1\% und Über das Internet deutsche/n GesprächspartnerIn...: 5,5\%). Die Mehrzahl der Nennungen (23,5\%) favorisiert demnach eine "Seminar «form, die methodisch sowohl frontal als auch mit Gruppenarbeitsphasen etc. stattfinden kann. Insgesamt zeugen die gegebenen Antworten vom eindeutigen Wunsch der Studierenden nach eigenverantwortlichen Lernformen, autonomem Lernen und (Selbst-Er-) Forschen sowie persönlichen Kontakten zu Deutschen: »Documentandome sintensiv « " sich intensiv Unterlagen beschaffen/sich informieren.) und »A través de una persona alemana y por investigación independiente «. (Durch eine deutsche Person und durch unabhängige Forschung) oder 
Conocer a un alemán y que éste me lo explica. (Eine/n Deutsche/n kennenlernen, so daß er/sie es mir erklärt.)

Ein besonders wichtiges Ziel ist wieder der Auslandsaufenthalt mit seinen Möglichkeiten zu authentischen Erfahrungen vor Ort, die die eigene Perspektive erweitern sollen: »Viajar a Alemania y estudiarlo >in sitio«". (Nach Deutschland reisen und dort vor Ort studieren/kennenlernen.) und "En Alemania, así podría estudiarlo desde un punto de vista alemán«. (In Deutschland, so könnte ich aus deutscher Perspektive studieren/lernen.) und »Ir a Alemania a investigar por mi cuenta «. (Nach Deutschland gehen und auf eigene Kosten/nach eigenem Interesse forschen.) Konkret wurde von einer Befragten auch Unterstützung bei der Orientierung durch die Dozentinnen und Dozenten gefordert: "Que se aconsejaran en clase libros haciendose introducciones en clase«. (Es sollten im Unterricht Bücher empfohlen werden, die im Seminar vorgestellt werden). Hier zeigt sich wieder der Bedarf an landeskundlichem Überblickswissen, das von den Studierenden als Angebot wahrgenommen werden kann, sich nach einer Orientierungsphase ihren Interessen folgend tiefer mit Einzelthemenbereichen (innerhalb der Breite landeskundlicher Wissensgebiete) zu beschäftigen.

Mit Frage 12 wird einerseits nach der Autorität gefragt, die landeskundlicher Unterricht im Heimatland besitzt. Andererseits wird auch um die Nennung von Inhalten der gewünschten authentischen Erfahrung im Zielsprachenland gebeten: Meinst Du, daß es bestimmte Themen gibt, über die man nur in Deutschland etwas erfahren kann? Welche? (¿Consideras que hay algunos temas sobre los que sólo se puede aprender en Alemania? ¿Cuáles?) Das Ergebnis auf diese Frage war überwältigend. Die befragten Studierenden nannten eine solche Flut von Themenbereichen, daß diese nach einem Schema von Byram/ Morgan (1994) bestimmten Gebieten zugeordnet werden mußten (siehe Anhang). Eindeutig lagen die Nennungen auch hier im »Kultur Zwei«-Bereich (nach Hofstede 1997): »el idioma « (die Sprache), »usos y costumbres« (Bräuche und Gewohnheiten) und »la conviviencia con alemanes" (Das Zusammenleben mit Deutschen). Die ergänzenden Bemerkungen der befragten StudentInnen machten wieder deutlich, wie stark die heutige Mobilitiät feststehender Teil des Fremdsprachenstudiums ist: »Yo creo que para aprender cualquiera cosa sobre un país extranjero hay que aprenderlo en él, pero hay que dominar su idioma«. (Ich glaube, um irgendetwas über ein Land zu lernen, muß man es dort lernen. Aber man muß seine Sprache beherrschen.). Eine andere Studentin meint: "Si, en mi opinión, el caracter de un pueblo sólo se puede conocer conviviendo en ese pueblo en este caso el alemán«. (Ja, meiner Meinung nach kann man ein Volk nur kennen(lernen), indem man mit diesem Volk zusammenlebt, in diesem Fall dem deutschen.) Der Unterschied zwischen touristischem Erleben einer Kultur und in einer Kultur leben wird von den Studierenden erkannt: »Creo que para tener una idea amplia, se necesita más tiempo sea en España o en Alemania«. (Ich glaube, daß man mehr Zeit braucht, um sich eine breite Vorstellung [von der Fremdkultur, E. F. B.] zu machen, egal ob in Spanien oder in Deutschland). Einzelne Nennungen reduzierten ihr landeskundliches Interesse allerdings ausschließlich auf die Sprachkenntnisse: »En mi opinión para lo único que es necesario estar en Alemania para aprender algo es el idioma ya que hay la información esta en todos lados". (Meiner Meinung nach ist das einzige, für das es notwendig ist, in Deutschland zu sein, die Sprache zu lernen, weil Informationen ja schon überall $\mathrm{zu}$ finden sind). 


\subsubsection{Wirkung der landeskundlichen Lehre} auf das Studium

Im letzten Teil der Umfrage interessierte die Funktion, die Landeskundeunterricht innerhalb der Fremdsprachenlehre hat.

Frage 13: Hast Du den Eindruck, daß sich Dein Interesse in Bezug auf Deutschland in den letzten Jahren verändert hat? (Ja/NeinAntwort) Wenn Du möchtest, erkläre die Gründe. (¿Tienes la impresión de que tu interés por Alemania ha cambiado en los últimos años? (Si/No-Respuesta) Sí quieres, explica los motivos) Das Ergebnis auf diese Frage war eindeutig positiv: für 76,5\% aller Befragten hat sich ihr Bezug zu Deutschland im Laufe ihres Sprach- und Landeskundeunterrichts verändert. Mit zunehmender Information/Orientierung steigt auch die Motivation für den Fremdsprachenerwerb. Die durchschlagende Wirkung der ERASMUS und SOKRATES-Aufenthalte in deutschsprachigen Ländern wurde von den befragten StudentInnen deutlich formuliert: »Si, desde que fui«. (Ja, seit ich dort war). Andere Studierende schrieben: "Si, cada vez me interesa más esa cultura casi desconocida para mi « (Ja, jedes Mal interessiert mich diese Kultur mehr, die mir fast unbekannt war/ist.). Und »Ha aumentado porque cuanto más me doy cuenta de lo que me falta por aprender «. ([Mein Interesse, E.F.B.] ist gewachsen, je mehr ich mir darüber klar werde, wieviel ich noch zu lernen habe.). Sowohl Deutschlandstereotype als auch authentische Erfahrungen im Zielsprachenland haben für die Studierenden motivierenden Charakter: " $\mathrm{Si}$, porque me parece un pueblo muy trabajador e interesante por tanto su historia« (Ja, weil es mir ein sehr fleißiges Volk $\mathrm{zu}$ sein scheint und wegen seiner Geschichte interessant ist) und " $\mathrm{Al}$ conocer personas alemanes me interesé más por su cultura«. (Durch das
Kennenlernen von Deutschen interessierte ich mich mehr für die Kultur).

Am Schluß der Umfrage sollte mit Frage 14 die Wirkung des modernen DACHKonzepts, das die Landeskundediskussion im Inland in den 80er Jahren bestimmte, von den Studierenden erfragt werden: Wenn $D u$ wählen könntest, wo würdest Du am liebsten etwas über deutsche Landeskunde (Cultura Alemana) lernen/studieren (unabhängig vom Geld)? (Si pudieras elegir, ¿dónde estudiarías Cultura Alemana (independientemente del dinero)?) Gegeben waren hier die Ländernamen Spaniens, Deutschlands, Österreichs, der Schweiz und Amerikas (als unbestimmtes Beispiel einer aktiven Auslandsgermanistik). Die Befragten hatten auch hier die Möglichkeit, eine freie Antwort zu ergänzen: In einem anderen Land, nämlich

Das Ergebnis zeigt, daß der Bezug zu Deutschland das Landeskundekonzept in Spanien stark dominiert: $82,7 \%$ der befragten Studierenden wünschen sich landeskundliche Studien in Deutschland, nur 24 Studierende $(7,7, \%)$ einen Aufenthalt in Österreich und nur $14(=4,5 \%)$ einen Aufenthalt in der Schweiz. Nur 3,5\% der Befragten bevorzugten landeskundlichen Unterricht in Spanien. Eine mögliche Erklärung für den Wunsch nach landeskundlichen Studien über ein fremdes Land innerhalb des eigenen Studiensystems könnte folgende Anmerkung signalisieren: »Es una pregunta difícil ya que hay temas problemáticas que creo que en Alemania y Austria se intentan evadir por lo que sería mejor estudiarlo en España«. (Das ist eine schwierige Frage, weil es problematische Themen gibt, die, glaube ich, in Deutschland und Österreich vermieden werden. Deshalb wäre es besser, sie in Spanien zu studieren). Ein gewisser Ideologieverdacht scheint also bis heute unter spanischen Studierenden einer Philologie zu 
existieren, wobei die Neugier auf und das Interesse an Deutschland eindeutig überwiegt.

Eindeutig an dem Ergebnis ist auch, daß der moderne Landeskundeansatz DACH bzw. DACHL die spanischen Universitäten (noch nicht) erreicht hat - entsprechend der Vorgabe des Ministerio de Educación i Deporte (MEC), die nur die Formulierung Historia y Cultura Alemana kennt. Auf der Suche nach Erklärungen für die teilweise ortsgebundene Affinität $\mathrm{zu}$ einem bestimmten Land (an der Universidad de Valencia kreuzten immerhin 5 von 19 Befragten Österreich als bevorzugtes Zielsprachenland an), schien die Erklärung plausibel, daß dort verhältnismäßig viele Lehrkräfte aus Österreich stammen und die nationale Herkunft der an den jeweiligen Universitäten tätigen Lehrkäfte für die Studieninteressen eine bedeutende Rolle spielt: Landeskundeunterricht im Ausland ist immer besonders stark von der persönlichen Einflußnahme der jeweiligen Lehrkraft abhängig.

\section{Zusammenfassung der Ergebnisse und Ausblick}

Die hier vorgestellte Studie zeigt, daß die Studierenden trotz der schwierigen Position landeskundlicher Lehre innerhalb des Philologiestudiums an spanischen Universitäten recht genaue Vorstellungen davon haben, was sie im Landeskundeunterricht lernen wollen und was nicht. Ihr landeskundliches Interesse geht dabei von ihren natürlichen Interessen aus und orientiert sich an einem Konzept des sprachlichen Handelns in der fremden Kultur. Wichtigstes Ziel ist die Vorbereitung auf einen Auslandsaufenthalt, für den alltagskulturelle Informationen (usos y costumbres) sowie die Fähigkeit zur interkulturellen Kommunikation ausschlaggebend sind. Erst an zweiter Stelle steht für sie der
Wissenserwerb, der vorwiegend auf historisches Wissen konzentriert ist und der ideologie- sowie kommerzfrei der Orientierung innerhalb der modernen Informationsflut dienen soll (Überblickswissen). Wie die befragten Studierenden mitteilen, spielen Lernmethoden eine ebenso wichtige Rolle wie Lerninhalte, da langfristig ein autonomer Umgang mit der fremden Kultur und seinen authentischen Zeugnissen angestrebt wird.

Diese Bedarfsmeldung der Studierenden wirkt neben dem Kultur- und Bildungskonzept des Ministerio de Educación $i$ Deporte (MEC) (und damit den Studienplänen der einzelnen Universitäten) für den Fremdsprachen- und Landeskundeunterricht wie Feuer und Wasser: es handelt sich um zwei unterschiedliche Kulturbegriffe. Nach Hofstede (1997) läßt sich das ministerielle Konzept dem Prinzip der »Kultur Eins« zuordnen, das sich

"aus dem lateinischen Ursprung ableitet, der das Bestellen des Bodens bezeichnet. In den meisten westlichen Sprachen bedeutet >Kultur gemeinhin >Zivilisation > oder >Verfeinerung des Geistes< und insbesondere die Ergebnisse dieser Verfeinerung, wie Bildung, Kunst und Literatur « (Hofstede 1997: 3).

Von den Studierenden wird jedoch eine ganz andere Ebene kulturellen Wissens gefordert, die der »Kultur Zwei«-Definition Hofstedes (1997) entspricht. Abgeleitet aus seiner lateinischen Wurzel meint es »Kultur als mentale Software», die sich

»auf eine viel weiter gefaßte, unter Sozialanthropologen übliche Bedeutung des Wortes [bezieht ...]. Sozial- (oder Kultur-) Anthropologie ist die Wissenschaft von den menschlichen Gesellschaften, insbesondere (aber nicht ausschließlich) den traditionellen oder >primitiven <. [...] Politiker und Journalisten verwechseln gelegentlich Kultur Eins und Kultur Zwei, ohne sich 
dessen bewußt zu sein. Anpassungsprobleme von Einwanderern in ihrem Aufnahmeland werden auf der Ebene der Förderung von Folkloregruppen diskutiert. Kultur Zwei bezieht sich jedoch auf wesentlich grundlegendere menschliche Prozesse als Kultur Eins; sie betrifft Dinge, die verletzen.« (Hofstede 1997: 4)

Auch in traditionellen Landeskundekonzepten wie dem spanischen scheinen $»$ Kultur Eins«-Konzepte nicht von dem der »Kultur Zwei« differenziert zu werden.

Die Schwierigkeiten der spanischen Germanistik mit dem aus Deutschland importierten Begriff »Landeskunde«, der in der spanischen Übersetzung cultura y civilización oder historia y cultura seine Wortkonnotation ändert, sind unübersehbar. Es geht darum, überfüllte Studienpläne in den Philologien von ihrem bildungsbürgerlichen Wissenskanon zu befreien und der »wachsenden Nachfrage nach kulturraumbezogenen Kompetenzen auf dem Arbeitsmarkt « (Lüsebrink 1999: 274) mit einem realistischen Ausbildungsprogramm $\mathrm{zu}$ entsprechen. Dazu gehört auch, daß die spanische Germanistik die schwierigen Berufsperspektiven ihrer Studierenden angesichts der schwachen Stellung des Deutschen als Schulfach im spanischen Bildungswesen eingesteht und - wie andere romanistische Studiengänge - mit neuen landeskundlich-kulturwissenschaftlichen Konzepten darauf reagiert (vgl. z. B. das Konzept der Universität Saarbrücken, Lüsebrink 1999).

Dazu gehört als erster Schritt eine »Entkanonisierung « (Lüsebrink 1999: 281) der traditionellen Landeskundelehre sowie die Öffnung der sog. "globalisierten« Landeskunde zu einer »integrierten «, »in der weite Bereiche kulturraumbezogener landeskundlicher Lehre und Forschung « nicht innerhalb der Germanistik, "sondern durch Nachbardisziplinen abge- deckt werden « (Lüsebrink 1999: 277). Das ist ein Vorschlag, der von Jané Carbó (1981) bereits in den 80er Jahren gemacht wurde.

Wichtig scheint auch eine längst fällige Aufarbeitung der unterschiedlichen Deutungsmuster von cultura/civilización und dem deutschen Kulturbegriff, die z. B. seit den 90er Jahren in der französischen Romanistik fester Bestandteil der Landeskundediskussion ist. (z.B. Thoma 1995; Röseberg 2001) Erst damit kann die theoretische Grundlage für eine eigenständige Landeskundeforschung in Spanien geschaffen werden, die von den spanischen Lehrbedingungen ausgehend Vorschläge für mögliche Curricula erarbeitet, didaktische Materialien zu sozialwissenschaftlichen und kulturanthropologischen Fragen erstellt etc. Dringend notwendig ist auch die Erstellung spanischsprachiger, kontrastiver Überblicksmaterialien zur Einführung in wichtige Themenbereiche der deutschsprachigen Gegenwartsgesellschaften, wie z. B. das deutsche/schweizer/österreichische Mediensystem, die Geschichte der Ausländerpolitik (von der viele spanische Familien im engeren oder weiteren Sinne betroffen waren/ sind und die das Deutschland-Image geprägt hat), Entwürfe zu Unterrichtseinheiten über Stereotypenbildung statt »Volksgeist«-Theorien (vgl. Marizzi 2000: 106) etc. Das Ziel landeskundlicher Lehre sollte sein, die Studierenden praxisnah und handlungsorientiert auf ihre Auslandserfahrungen vorzubereiten, sie theoretisch und anhand ausgewählter Beispiele (z. B. der Werbesprache, Wirtschaftskommunikation, Filmtechnik etc.) kontrastiv in fortgeschrittene Phasen interkultureller Kompetenz einzuführen (vgl. Roche 2001: 50 ff.) und damit erst Grundlagen für eine fruchtbare interkulturelle Kommunikation innerhalb der EU zu legen. 


\section{Anhang: Fragebogen zum Landeskunde-Unterricht an spanischen Universitäten}

Liebe/r Studierende,

mit diesem Fragebogen soll versucht werden, das Interesse an landeskundlichen Inhalten zu evaluieren. Bitte antworte bei den Fragen spontan und entsprechend Deinen persönlichen Interessen oder Erfahrungen. Wir würden gerne wissen, was Dich an Deutschland interessiert oder welche Wissensbereiche Dir bei bisherigen Kursen/Seminaren fehlten/ fehlen.

1.) Was studierst Du im Hauptfach

Ich studiere Deutsche Philologie

Ich studiere Übersetzen und Dolmetschen

Ich belege den Kurs

Ich studiere/lerne eine andere Philologie (welche?)

2.) In welchem Studienjahr bist Du?

Seit wann lernst/studierst Du Deutsch?

3.) Dieser Kurs (»Deutsche Landeskunde«) ist innerhalb Deines Studiums:

obligatorisch (Pflichtfach) (troncal) O obligatorisch (obligatorio)

für HörerInnen aller Fakultäten (libre elección)

○

4.) Für welche der folgenden Themen interessierst du Dich im allgemeinen?

(Du kannst bis zu 5 Elemente ankreuzen!)
O Mode
Kunst/Photographie
Fachliteratur über
O Popmusik/moderne
O Politik Musik
Olassische Musik
(Massen)Medien
O Sport
O Informatik
O Kino
O Familie
O Geschichte
O Essen
O Tourismus/Reisen
O Bräuche/Gewohn- heiten
Berufliche Weiterbil- dung
O Parteien
O Museen
$\bigcirc$ andere, nämlich
O Die Europäische Union
O Gewerkschaften
O Geographie

5.) Welche der folgenden Themen interessieren Dich in Bezug auf Deutschland? (auch hier sind bis zu 5 Kreuzchen möglich!)
O Mode
O Kunst/Photographie
Fachliteratur über
Popmusik/moderne
O Politik Musik
klassische Musik
O (Massen)Medien
O Sport
Informatik
O Kino
O Familie
Geschichte
O Essen
O Tourismus/Reisen
O Bräuche/Gewohn- heiten
O Berufliche Weiterbil- dung
O Parteien
O Museen
O Literatur/Romane
O Die Europäische Union
O Gewerkschaften
$\bigcirc$ andere, nämlich
O Geographie 
6.) Hattest oder hast Du persönlichen oder familiären Kontakt zu/nach Deutschland?

(Bitte markiere nicht mehr als 2 Antworten)

O Ja, Teile meiner Familie leben in $O$ Ich hatte früher eine/n Brieffreund/in Deutschland. aus Deutschland.

Ja, ich habe Freunde in Deutschland, die ich regelmäßig sehe.

Ja, ich war einige Male in Deutschland, aber jetzt habe ich keinen Kontakt ○ mehr zu den Leuten, die ich kannte.

Nein, ich war noch nie in Deutschland und kenne auch keine Deutschen persönlich.

Ich kenne die Deutschen vom Sehen auf der Straße oder am Strand.

7.) Erinnerst Du Dich, ob Du im Sprachunterricht oder in der Uni schon einmal gedacht hast, daß Du gerne mehr über ein bestimmtes Thema wissen möchtest? Erinnerst Du Dich, zu welchem Bereich dieses Thema gehörte? (Bitte nicht mehr als 3 Themengebiete ankreuzen!)

\begin{tabular}{l|l}
\multicolumn{1}{c|}{ über Spanien } & \multicolumn{1}{c}{ über Deutschland } \\
\hline O Politik & O Politik \\
\hline O Geographie & O Geographie \\
\hline O Geschichte & O Geschichte \\
\hline O Wirtschaft & O Wirtschaft \\
\hline O Kunst & O Kunst \\
\hline O Musik & O Musik \\
\hline O Gesellschaft & O Gesellschaft \\
\hline O Essen & O Essen \\
\hline O Bräuche/Gewohnheiten & O Bräuche/Gewohnheiten
\end{tabular}

8.) Um neue Informationen über ein Thema (egal welches) zu erhalten, welchem öffentlichen Medium glaubst Du am meisten? (bezüglich der Glaubwürdigkeit der vermittelten Information, z. B. bei den Anschlägen in den USA) (Bitte nicht mehr als 3 Kreuze machen!)
O dem TV
$O$ der Zeitung
O dem Radio
meiner Zeitung, die ich immer kaufe
$O$ einem Buch von einer $/ \mathrm{m}$ bekannten AutorIn

einer Statistik

einer Fachzeitschrift über das Thema

$O$ einem wissenschaftlichen Fachbuch
Ich glaube es nur, wenn die Informa- tion bei verschiedenen Medien über- einstimmt.

9.) Welche historischen Epochen interessieren Dich am meisten?

(Bitte nicht mehr als 3 Elemente ankreuzen)
O Das Mittelalter
Der 1. Weltkrieg
O Die Reformation
O Die Weimarer Republik
Das 18. Jahrhundert insgesamt
O Der Nationalsozialismus
O Die Romantik
O Der 2. Weltkrieg
Das 19. Jahrhundert insgesamt
O nach 1945
O Der Realismus
O La transición
O Die Gründung des 3. Reichs
nach dem Francismo
O Das 20. Jahrhundert insgesamt
O Ereignisse seit 1990 
10.) In spanischen Zeitungen gibt es viele Informationen über Deutschland und die deutsche Politik. Interessieren Dich diese Artikel? Warum? Warum nicht?

11.) Wenn Du Dir ein Thema über Deutschland aussuchen könntest, wie würdest Du am liebsten etwas darüber erfahren? (Bitte nicht mehr als 2 Antworten ankreuzen)

O Ein spezielles Seminar darüber belegen.

Einen Vortrag/eine Konferenz von einem Spezialisten/einer Spezialistin darüber hören.

Eine allgemeine Einführung in einem Überblicksseminar erhalten (neben anderen Themen).

In einer Arbeitsgruppe Informationen über das Thema sammeln und sich dann über das erhaltene Wissen austauschen.

O Selbständig das Thema erforschen und eine schriftliche Hausarbeit darüber schreiben.

Ein Buch darüber lesen.

O Ein Semester freinehmen und mehrere Bücher darüber lesen.

Ö̈ber das Internet einen deutschen Gesprächspartner/eine Gesprächspartnerin O suchen, um mit ihr/ihm über das Thema zu sprechen.

12.) Meinst $\mathrm{Du}$, daß es bestimmte Themen gibt, über die man nur in Deutschland etwas erfahren kann? Welche?

13.) Hast Du den Eindruck, daß sich Dein Interesse in Bezug auf Deutschland in den letzten Jahren verändert hat?

$\bigcirc$ ja $O$ nein

wenn Du möchtest, erkläre die Gründe:

14.) Wenn Du wählen könntest, wo würdest Du am liebsten etwas über deutsche Landeskunde (cultura alemana) lernen/studieren (unabhängig vom Geld)?
$O$ in Spanien
$O$ in Deutschland
$O$ in Österreich
$O$ in der Schweiz
$O$ in Amerika
$O$ in einem anderen Land, nämlich

Auswertung der Frage 12: Meinst Du, daß es bestimmte Themen gibt, über die man nur in Deutschland etwas erfahren kann? Welche?

\begin{tabular}{||c|c|c||}
\hline $\begin{array}{c}\text { Themen- } \\
\text { bereiche/temas }\end{array}$ & \multicolumn{1}{|c||}{$\begin{array}{c}\text { areas of study } \\
\text { (Byram/Morgan et al. (1994)) }\end{array}$} & $\begin{array}{l}\text { Weitere Nennungen der Be- } \\
\text { fragten, die diesem Themen- } \\
\text { bereich zuzuordnen sind }\end{array}$ \\
\hline $\begin{array}{c}\text { el idioma } \\
\text { (die Sprache) }\end{array}$ & $\begin{array}{l}\text { Social interaction: conventions of verbal } \\
\text { behaviour in social interaction at differ- } \\
\text { ing levels of familiarity, as outsider and } \\
\text { insider within social groups. }\end{array}$ & $\begin{array}{l}\text { giros linguisticos, ciertas pa- } \\
\text { labras, expreciones, lenguage } \\
\text { coloquial, dialectos, la pre- } \\
\text { nunciación, el acento, refra- } \\
\text { nes, coletillas, bromas, hablar } \\
\text { coloquial, aprender bien la } \\
\text { lengua, tener una excelente } \\
\text { fluidad de idioma }\end{array}$ \\
\hline
\end{tabular}




\begin{tabular}{|c|c|c|}
\hline $\begin{array}{l}\text { Themen- } \\
\text { bereiche/temas }\end{array}$ & $\begin{array}{c}\text { areas of study } \\
\text { (Byram/Morgan et al. (1994)) }\end{array}$ & $\begin{array}{l}\text { Weitere Nennungen der Be- } \\
\text { fragten, die diesem Themen- } \\
\text { bereich zuzuordnen sind }\end{array}$ \\
\hline $\begin{array}{c}\text { la conviviencia } \\
\text { con alemanes } \\
\text { (Das Zusammen- } \\
\text { leben mit Deut- } \\
\text { schen) }\end{array}$ & $\begin{array}{l}\text { social interaction: conventions of non- } \\
\text { verbal behaviour in social interaction at } \\
\text { differing levels of familiarity, as outsider } \\
\text { and insider within social groups }\end{array}$ & $\begin{array}{l}\text { contacto cercano; gestos; los } \\
\text { comportamientos (porque no } \\
\text { son exportables); manera de } \\
\text { pensar }\end{array}$ \\
\hline $\begin{array}{c}\text { usos y } \\
\text { costumbres } \\
\text { (Gewohnheiten } \\
\text { und Gebräuche) }\end{array}$ & $\begin{array}{l}\text { belief and behaviour: routine and taken- } \\
\text { for-granted actions within a social group } \\
\text { - national or sub-national - and the } \\
\text { moral and religious beliefs which are } \\
\text { embodied within them; secondly, rou- } \\
\text { tines of behaviour taken from daily life } \\
\text { which are not seen as significant markers } \\
\text { of the identity of the group }\end{array}$ & $\begin{array}{l}\text { los valores culturales; forma } \\
\text { de vivir; su manera de vivir; } \\
\text { modo de vivir; vida coti- } \\
\text { diana; ideales; los referentes } \\
\text { al modo de ser y ver el } \\
\text { mundo de aquella gente; lo } \\
\text { que esta mal visto/bien visto } \\
\text { etc. }\end{array}$ \\
\hline $\begin{array}{l}\text { el caracter de los } \\
\text { alemanes } \\
\text { (Der deutsche } \\
\text { Charakter) }\end{array}$ & $\begin{array}{l}\text { social identity and social groups: groups } \\
\text { within the national-state which are the } \\
\text { basis for other than national identity, } \\
\text { including social class, regional identity, } \\
\text { ethnic minority, professional identity, } \\
\text { and which illustrate the complexity of } \\
\text { individuals'social identities and of a na- } \\
\text { tional society [...] }\end{array}$ & $\begin{array}{l}\text { la mentalidad; lo que atañe el } \\
\text { caracter alemán }\end{array}$ \\
\hline $\begin{array}{c}\text { Sociedad } \\
\text { (Gesellschaft) }\end{array}$ & $\begin{array}{l}\text { social-political institutions: institutions } \\
\text { of the state - and values and meanings } \\
\text { they embody - which characterise the } \\
\text { state and its citizens and which consti- } \\
\text { tute a frame-work for ordinary, routine } \\
\text { life within the national and sub-national } \\
\text { groups: provision for health-care, for law } \\
\text { and order, for social security, for local } \\
\text { government, etc. }\end{array}$ & $\begin{array}{l}\text { la organisación social; situa- } \\
\text { ción económico real; proble- } \\
\text { mas sociales }\end{array}$ \\
\hline $\begin{array}{l}\text { Folklore } \\
\text { (Folklore) }\end{array}$ & $\begin{array}{l}\text { national cultural heritage: cultural arte- } \\
\text { facts perceived to be emblems and em- } \\
\text { bodiments of national culture from past } \\
\text { and presence; in particular those which } \\
\text { are »known« to members of the nation }\end{array}$ & $\begin{array}{l}\text { fiestas populares; gastro- } \\
\text { nomía; comida, platos tipicos; } \\
\text { arte }\end{array}$ \\
\hline $\begin{array}{c}\text { tratamiento } \\
\text { familiar } \\
\text { (Familiäre } \\
\text { Umgangsweisen) }\end{array}$ & $\begin{array}{l}\text { socialisation and the life-cycle: institu- } \\
\text { tions of socialisation - families, schools, } \\
\text { employment, religion, military service- } \\
\text { and the ceremonies which mark passage } \\
\text { through stages of social life; representa- } \\
\text { tion of divergent practices in different } \\
\text { social groups a well as national auto- } \\
\text { stereotypes of expectations and shared } \\
\text { interpretations }\end{array}$ & habitos de la vida familiar \\
\hline
\end{tabular}




\begin{tabular}{||c|l|l||}
\hline $\begin{array}{c}\text { Themen- } \\
\text { bereiche/temas }\end{array}$ & \multicolumn{1}{|c|}{$\begin{array}{c}\text { areas of study } \\
\text { (Byram/Morgan et al. (1994)) }\end{array}$} & $\begin{array}{l}\text { Weitere Nennungen der Be- } \\
\text { fragten, die diesem Themen- } \\
\text { bereich zuzuordnen sind }\end{array}$ \\
\hline $\begin{array}{c}\text { historia/nacismo } \\
\text { (Geschichtel } \\
\text { National- } \\
\text { sozialismus) }\end{array}$ & $\begin{array}{l}\text { national history: periods and events, } \\
\text { historical and contemporary, which are } \\
\text { significant in the constitution of the na- } \\
\text { tion and its identity - both actually sig- } \\
\text { nificant and, not necessarily identical, } \\
\text { perceived as such by its members }\end{array}$ & $\begin{array}{l}\text { la repercusión (Nachwir- } \\
\text { kung) de nacismo en la perso- } \\
\text { nalidad alemana; la 2. guerra } \\
\text { mundial, immigración }\end{array}$ \\
\hline $\begin{array}{c}\text { Geografia } \\
\text { (Geographie) }\end{array}$ & $\begin{array}{l}\text { national geography: geographical fac- } \\
\text { tors within the nation boundaries which } \\
\text { are significant in members' perceptions } \\
\text { of their country; other factors which are } \\
\text { information (known but not significant } \\
\text { to members) essential to outsiders in } \\
\text { intercultural communication [...] }\end{array}$ & geografía \\
\hline \hline
\end{tabular}

\section{Literatur}

Bachmann, Saskia: »Kontrastive Landeskunde«. In: Filología Alemana y Didáctica del Aleman/Germanistik und Deutschunterricht. Materialien des V. Symposiums des Spanischen Deutschlehrerverbandes; Valladolid 6.-10. November 1989. Hrsg. von Regales Serna, Antonio. Universidad de Valladolid, 1992, 311-321.

Blanco-Camblor, $\mathrm{M}^{\mathrm{a}}$ Luz: »Historia y Cultura Alemanas «, ¿Una signatura indispensable en los estudios de Germanística?. In: Lengua, literatura y cultura alemanas ante el umbral del nuevo Milenio, Actas de la IX. Semana de Estudios Germánicos (30 de marzo - 2 de abril de 1998). Hrsg. von Acosta, Luis A.; Hernández, Maria Isabel; Wittenberg, S. Vol. I. Madrid: Ediciones del Orto, 2000, 233-262.

Blanco-Camblor, $\mathrm{M}^{\mathrm{a}}$ Luz: »Los textos literarios como transmisores de conocimientos de >Landeskunder en clase de idiomas«, Estudios Filológicos Alemanes 1 (2002), 207-225 (Universidad de Sevilla).

Bosch Roig, Gloria: Sprachenpolitik und Deutschunterricht in Spanien. Diss., 1999. Im Internet publiziert unter: http://www. goethe.de/wm/mad/pdf/DaF-SP.pdf

Byram, Michael; Morgan, Carol and colleagues: Teaching-and-Learning Languageand-Culture. Cleverland: Multilingual Matters, 1994.

Decker, Thomas: Manual de Aleman para universitarios. Valencia: Editorial Unialemán, 2001.
Doyé, Peter: »Neuere Konzepte landeskundlichen Lernens«, Der Fremdsprachliche Unterricht - Englisch. Intercultural Language Learning, 26, 3 (1992), 4-7.

Geertz, Clifford: Dichte Beschreibung. Frankfurt/ M.: Suhrkamp, 1983.

Jané Carbó, Jordi: "Landeskunde im Sprachunterricht «. In: Akten des 1. Iberischen Germanistentreffens. Hrsg. von Pérez Varas, F.; Bujan Lopez, C. Salamanca: Ediciones Universidad de Salamanca, 1981, 257-270.

Jané Carbó, Jordi / Wolff, Jürgen: »Länderbericht Spanien, Deutsch - Sprache mit Zukunft?«, Info DaF 18, 2 (1991), 198-207.

Janés, Alfonsina: Historia de la cultura alemana. Barcelona: MAES S. C., 1992.

Fulbrook, Mary: Historia de Alemania. Cambridge: University Press, 1990. Übersetzung vom Englischen ins Spanische von Beatriz García Ríos, 1995.

Hofstede, Geert: Lokales Denken, globales Handeln. München: Beck, 1997.

Iberische Lektorenarbeitsgruppe der DAAD-Lektorinnen und Lektoren: »Eine Erhebung zum Deutschlandbild der Germanistikstudentinnen und -studenten auf der iberischen Halbinsel«, Info DaF 26, 4 (1999), 355-377.

Keim, Lucrecia: »Deutschunterricht und Germanistikstudium in Spanien«. In: Helbig, Gerd; Götze, Lutz; Henrici, Gert; Krumm, Hans-Jürgen (Hrsg.): Deutsch als Fremdsprache. Ein internationales Handbuch. 2. Halbband. New York u.a.: de Gruyter, 2001, 1516-1523. 
Knapp-Potthoff, Annelie: „Sprach(lern)bewußtheit im Kontext«. In: Henrici, Gert; Zöfgen, Ekkehard (Hrsg.): Fremdsprachen lehren und lernen. Tübingen: Narr, 1997, 923.

Koreik, Uwe / Henrici, Gert (Hrsg.): »Zur Konstituierung des Fachs Deutsch als Fremdsprache", Deutsch als Fremdsprache: wo warst Du?, wo bist Du?, wohin gehst Du? Schneider: Baltmannsweiler, 1980, 142.

Kramsch, Claire J.: »Bausteine zu einer Kulturpädagogik des Fremdsprachenunterrichts «, Jahrbuch Deutsch als Fremdsprache, 17 (1991), 104-120.

Lüsebrink, Hans-Jürgen: »)Kulturraumbezogene Landeskunde< und >Kulturwissenschaft< in der Romanistik - das Beispiel Saarbrücken«, Jahrbuch Deutsch als Fremdsprache 25 (1999), 273-288.

Marizzi, Bernd: »Bemerkungen zur Darstellung der deutschsprachigen Literatur in spanischen Schulliteraturgeschichten des 20. Jahrhunderts«. In: Lengua, literatura y cultura alemanas ante el umbral del nuevo Milenio, Actas de la IX. Semana de Estudios Germánicos (30 de marzo - 2 de abril de 1998). Hrsg. von Acosta, Luis A.; Hernández, Maria Isabel; Wittenberg, S. Vol. I. Madrid: Ediciones del Orto, 2000, 95-108.

Marizzi, Bernd: »Apuntes acerca de la historia de la enseñanza del alemán como lengua con fines específicos en España«. In: El alemán: una lengua extranjera en España. Hrsg. von Goethe Institut InterNationes/Instituto Alemán de Cultura Madrid (2002), 63-65.

Melde, Wilma: Zur Integration von Landeskunde und Kommunikation im Fremdsprachenunterricht. Tübingen: Narr, 1987.

Orduña, Javier: Wachsende Nachfrage nach der Lehre der deutschen Sprache an katalanischen Privatschulen. Unveröffentlichtes Manuskript, 2002.

Palau-Ribes Casamitjana, Francisca: »Die Rezeption der deutschen Sprache in Spanien. Rückblick und Ausblick «. In: Akten des 1. Iberischen Germanistentreffens. Hrsg. von Pérez Varas, F.; Bujan Lopez, C. Salamanca: Ediciones Universidad de Salamanca, 1981, 95-104.

Penning, Dieter: "Landeskunde als Thema des Deutschunterrichts - fächerübergreifend und/oder fachspezifisch? «, Info DaF 22, 6 (1995), 626-640.
Picht, Robert: »Interesse und Vergleich: zur Sozialpsychologie des Deutschlandbilds«, Jahrbuch Deutsch als Fremdsprache 6 (1980), 120-132.

Regales, Antonio: "Germanistik und Deutsch als Fremdsprache in Spanien. Betrachtungen im historischen Kontext «. In: Götze, L. (Hrsg.): Schriften zur Deutsch Didaktik: Deutsch als Fremdsprache. Situation eines Fachs. Bonn-Bad-Godesberg: Dürr, 1987, 60-73.

Regales, Antonio: »Über Landeskunde in spanischen Lehrbüchern für Deutsch als Fremdsprache", Neusprachliche Mitteilungen aus Wissenschaft u. Praxis 1990, 247-253.

Regales, Antonio: »Pacifismo y >Landeskunde«". In: Filología Alemana y Didáctica del Aleman/Germanistik und Deutschunterricht, Materialien des V. Symposiums des Spanischen Deutschlehrerverbandes; Valladolid 6.-10. November 1989. Hrsg. von Regales Serna, Antonio. Universidad de Valladolid, 1992, 337-367.

Roche, Jörg: Interkulturelle Sprachdidaktik. Tübingen: Narr, 2001.

Röseberg, Dorothee: Kulturwissenschaft Frankreich. Stuttgart: Klett, 2001.

Saalbach, Mario: »DaF auf den Kopf gestellt«, Info DaF 26, 4 (1999), 327-347.

Sainz Lerchundi, Asunción; Sánchez Hernández, Julio: Vocabulario de cultura alemana. Sevilla: Kronos, 1997.

Soliño Pazó, Mar: »Orientierungen zu einer neuen Perspektive der deutschen Sprachwissenschaft in der Auslandsgermanistik«. In: Lengua, literatura y cultura alemanas ante el umbral del nuevo Milenio, Actas de la IX. Semana de Estudios Germánicos 1998. Hrsg. von Acosta, Luis A.; Hernández, Maria Isabel; Wittenberg, S. Vol. II. Madrid: Ediciones del Orto, 2000, 93-104.

Thoma, Heinz: »Macht und Ohnmacht von Deutungsmustern, Civilisation/Kultur Culture/Zivilisation«. In: Lüsebrink, H.Jürgen; Röseberg, Dorothee (Hrsg.): Landeskunde und Kulturwissenschaft in der Romanistik. Tübingen: Narr, 1995, 13-22.

Weinrich, Harald: »Forschungsaufgaben des Faches Deutsch als Fremdsprache«. In: Wierlacher, Alois (Hrsg.): Fremdsprache Deutsch. Bd. 1, München: Fink, 1980, 28-45.

Zimmermann, Daniel: "Hochschulpolitik in Spanien ", Forschung und Lehre 5 (2002) und Tranvia 66 (2002), 55-57. Im Internet unter: http://pagina.de/daniel.zimmermann/ 